\title{
CATEGORICAL COMPACTNESS FOR RINGS
}

\author{
TEMPLE H. FAY and STEPHAN V. JOUBERT
}

(Received 22 June 1993; revised 25 May 1994)

\author{
Communicated by P. Schultz
}

\begin{abstract}
In this paper we study categorical compactness with respect to a class of objects $\mathscr{F}$ being motivated by examples arising from modules, abelian groups, and various classes of non-abelian groups. This theory is then applied to the category of not necessarily associative rings. In particular, we study the example arising from the class of all torsion-free rings. This work extends some recent results of B. J. Gardner for associative rings and radical classes.
\end{abstract}

1991 Mathematics subject classification (Amer. Math. Soc.): 16A21, 16A63, 18A20.

Keywords and phrases: torsion theory, semisimple, polynomial regularity, closure operator, categorical compactness.

In this paper we study categorical compactness with respect to a class $\mathscr{F}$ in the category of not necessarily associative rings, denoted NRng. In doing so, we also obtain results for other categories: alternative rings, AlRng; associative rings, ARng; groups, Grp; $R$-modules, R-Mod; and, of course, abelian groups, $\mathbf{A B}$. We mention these categories in particular, as these are the categories in which the interesting examples arise. Our preliminary results are quite general and hold in a wide variety of settings and one could assume to be working in a finitely complete, finitely cocomplete, quasi-additive category $\mathscr{C}$.

If $\mathscr{F}$ is an isomorphism closed class of objects, containing the zero object, and if $A$ is a subobject of $B$, we define the $\mathscr{F}$-closure of $A$ in $B$ by $\mathrm{Cl}_{\mathscr{B}}(A)=\bigcap\{I \mid A \subseteq I$, $I$ is normal in $B$ and $B / I \in \mathscr{F}$. In the case that $\mathscr{F}$ is closed under subobjects, this closure becomes an idempotent closure operator. We say $A$ is $\mathscr{F}$-closed (in $B$ ) provided $A$ is normal in $B$, and $B / A$ belongs to $\mathscr{F}$. A drawback to the definition of $\mathscr{F}$-closure given above is that $\mathrm{Cl}_{\mathscr{B}}(A)$ need not be $\mathscr{F}$-closed. However, in the case that $\mathscr{F}$ is closed under subdirect products, $\mathrm{Cl}_{\mathscr{B}}(A)$ is $\mathscr{F}$-closed. In ring theory,

(C) 1995 Australian Mathematical Society 0263-6115/95 \$A2.00+0.00 
semisimple classes and torsion-free classes are closed under subdirect products as are quotient-reflective classes, and it is these classes that lead to interesting examples.

In our first section, we study our more general notion of $\mathscr{F}$-closure, developing the necessary preliminary results. Naturally, the strongest results occur when the $\mathscr{F}$-closure becomes an idempotent closure operator. We also investigate various notions of denseness and separatedness (the categorical analogue of Hausdorffness), in particular, examining the class of objects $X$ having a closed diagonal in $X \times X$. Section 2 is devoted to the notion of $H_{\mathscr{F}}$-closed which is a generalization of the notion of absolutely closed developed in [4]. Categorical compactness with respect to the class $\mathscr{F}$ is developed in Section 3. Here we show that many of the basic results for categorically compact objects hold despite $\mathrm{Cl}_{\mathscr{F}}(-)$ not being a closure operator.

The main Section 4 concerns applying this theory in the categories NRng and ARng. We improve slightly the results of Gardner [11]. For any class $\mathscr{F}$, if $R$ has an identity, then $R$ is $\mathscr{F}$-compact. For ARng, if $\mathscr{F}$ is semisimple and consists solely of semiprime rings and is closed under ideals, then every ring is $\mathscr{F}$-compact. More interestingly, we consider the example arising from taking $\mathscr{F}_{\tau}$ to be the class of all rings whose underlying additive group is torsion-free. The class $\mathscr{F}_{\tau}$ is closed under subrings, products, and extensions. This gives rise to a non-trivial class of $\mathscr{F}_{\tau}$-compact rings with interesting properties. There are rings which are $\mathscr{F}_{\tau}$-compact, but whose additive groups are not categorically compact with respect to torsion-free groups in the category of abelian groups. However, any ring whose additive group is $\mathscr{F}_{\tau}$-compact as a group turns out to be $\mathscr{F}_{\tau}$-compact as a ring. The analogues of compact subspaces of Hausdorff spaces being closed, and closed subspaces of compact spaces being compact, both fail here. These analogues do hold, however, for categorical compactness relative to a hereditary torsion theory for modules [5]. Furthermore, these examples show that while $H_{\mathscr{F}}$-closed objects are always $\mathscr{F}$ compact, the converse is false.

\section{Closure, denseness, and separatedness}

Throughout this paper, we let $\mathscr{F}$ denote an isomorphically closed class of $\mathscr{C}$-objects containing 0 . We call a subobject $A$ of $B \mathscr{F}$-closed provided $A$ is normal in $B$ and $B / A$ belongs to $\mathscr{F}$. Similarly, we define the $\mathscr{F}$-closure of a subobject $A$ in $B$ by setting $\mathrm{Cl}_{\mathscr{B}}(A)$ to be the intersection of all $\mathscr{F}$-closed subobjects of $B$ containing $A$. We use the notation $\tau_{\mathscr{F}} B$, or more simply $\tau B$ when the class $\mathscr{F}$ is understood, to denote $\mathrm{Cl}_{B}(0)$. If $f: B \rightarrow C$ is a morphism enjoying the property that $\mathrm{Cl}_{C}(f(B))=C$, then $f$ is called $\mathscr{F}$-dense. This approach is similar to that taken in $[2,3]$ and $[5]$. where a closure operator on subobjects was obtained. However, we have here a slightly more general situation which sheds light on a number of results concerning closure 
operators of this type, as is seen by our first result. If $B$ is an arbitrary object and $A$ is a subobject, we write $A \leq B$.

THEOREM 1.1. For any class $\mathscr{F}$, if $A$ is a subobject of $B$, then:

(i) $A \leq \mathrm{Cl}_{B}(A) \leq B$;

(ii) for $A_{1} \leq A_{2} \leq B, \mathrm{Cl}_{B}\left(A_{1}\right) \leq \mathrm{Cl}_{B}\left(A_{2}\right)$;

(iii) $\mathrm{Cl}_{B}\left(\mathrm{Cl}_{B}(A)\right)=\mathrm{Cl}_{B}(A)$ (idempotency);

(iv) for every object $B, \tau(B / \tau B)=0$.

ProOF. The verifications of (i), (ii), and (iii) follow routinely from the definition of $\mathscr{F}$-closure. To verify (iv), let $\varphi: B \rightarrow B / \tau B$ be the natural map, let $\mathscr{A}=\{I \mid I$ is $\mathscr{F}$-closed in $B\}$, and let $\mathscr{B}=\{K \mid K$ is $\mathscr{F}$-closed in $B / \tau B\}$. For each $K \in \mathscr{B}$, the inverse image $\varphi^{\leftarrow}(K) \in \mathscr{A}$, and if $I \in \mathscr{A}$, then $\varphi^{\leftarrow} \varphi(I)=I$. Using the fact that inverse image and intersections commute, we have $\varphi^{\leftarrow}(\tau(B / \tau B))=\bigcap\left\{\varphi^{\leftarrow}(K) \mid K \in\right.$ $\mathscr{B}\}=\bigcap\{I \mid I \in \mathscr{A}\}=\tau B$. Thus $0=\varphi(\tau B)=\tau(B / \tau B)$.

It is a trivial observation that for any subobject $A$ of an object $B, \mathrm{Cl}_{B}(A)=$ $\mathrm{Cl}_{B}(\langle A\rangle)$, where $\langle A\rangle$ denotes the smallest normal subobject of $B$ containing $A$. By analogy with the terminology from universal algebra, a mapping which assigns to each object $B$ an ideal $\tau B$ is called a Hoehnke radical [14] provided for each onto homomorphism $f: B \rightarrow C, f(\tau B) \subset \tau(f(B))$ and moreover, $\tau(B / \tau B)=0$ for all $B$. It is clear that for any class $\mathscr{F}, \tau_{\mathscr{F}}(-)$ defines a Hoehnke radical which determines the $\mathscr{F}$-closure via the following lemma whose proof is similar to the proof of part (iv) just given.

LEMMA 1.2. If $A \leq B$, then $\varphi^{\leftarrow} \tau(B /\langle A\rangle)=\mathrm{Cl}_{B}(A)$.

The next result shows that under the relatively weak assumption of $\mathscr{F}$ being closed under subobjects, we have an idempotent closure operator in the usual sense (see $[3,2])$. This subsumes [11, Proposition 1.2].

THEOREM 1.3. If $\mathscr{F}$ is closed under formation of subobjects, then for a morphism $f: B \rightarrow C, f\left(\mathrm{Cl}_{B}(A)\right) \subset \mathrm{Cl}_{C}(f(A))$ (continuity condition); hence $\mathrm{Cl}(-)$ is an idempotent closure operator. Moreover, the composition of $\mathscr{F}$-dense maps is $\mathscr{F}$ dense.

ProOF. Let $K$ be an $\mathscr{F}$-closed subobject of $C$ containing $f(A)$. It follows that $B / f^{-} K$ is a subobject of $C / K$ and hence $f^{-} K$ is an $\mathscr{F}$-closed subobject of $B$ containing $A$. Letting $\mathscr{B}=\{K \mid f(A) \subset K$, and $K$ is $\mathscr{F}$-closed in $C\}$, it is clear that $\mathrm{Cl}_{B}(A) \subset \bigcap\left\{f^{\leftarrow} K \mid K \in \mathscr{B}\right\}=f^{\leftarrow}(\cap \mathscr{B})$, and thus $f\left(\mathrm{Cl}_{B}(A)\right) \subset \cap \mathscr{B}=\mathrm{Cl}_{C}(f(A))$.

Assume that $A \stackrel{e}{\rightarrow} B$ and $B \stackrel{f}{\rightarrow} C$ are $\mathscr{F}$-dense. If $K \in \mathscr{A}=\{K \mid f e(A) \subset K$, and $K$ is $\mathscr{F}$-closed in $C$ \}, then as $B / f^{\leftarrow} K$ is a subobject of $C / K$ as above, $f^{\leftarrow} K$ is 
$\mathscr{F}$-closed in $B$ and hence equals $B$ from the $\mathscr{F}$-denseness of $e$. Thus $f(B) \subset K$ and so $C=\mathrm{Cl}_{C}(f(B)) \subseteq K$, that is, $\mathscr{A}=\{C\}$. This means that $\mathrm{Cl}_{C}(f e(A))=C$, and that $f e$ is $\mathscr{F}$-dense.

There are several classes of objects determined by the class $\mathscr{F}$ that are useful. We define $\mathscr{T}_{h}(\mathscr{F})=\left\{A \mid \operatorname{Hom}_{\mathscr{C}}(A, F)=0\right.$, for all $\left.F \in \mathscr{F}\right\}, \mathscr{T}_{r}(\mathscr{F})=\{A \mid A$ has no non-zero images belonging to $\mathscr{F}\}$, and $\mathscr{T}_{n}(\mathscr{F})=\{A \mid f: A \rightarrow F, f(A)$ normal in $F$, $F \in \mathscr{F}$, implies $f=0$ \}. We shall delete the reference to the class $\mathscr{F}$ when there is little likelihood of confusion and refer, for example, simply to $\mathscr{T}_{h}$. Recall that a class $\mathscr{F}$ is called $h$-regular (respectively $n$-regular) provided that for $0 \neq G \leq F \in \mathscr{F}$ (respectively, $0 \neq G$ normal in $F$ ), there exists a $B \neq 0, B \in \mathscr{F}$, with $B$ an image of $G$. In particular, any class closed under subobjects is both $h$-regular and $n$-regular. Semisimple and torsion-free classes of rings are $n$-regular. The following proposition has a routine proof which is omitted.

PROPOSITION 1.4. For any class $\mathscr{F}$, the following hold:

(i) $\mathscr{T}_{h} \subset \mathscr{T}_{n} \subset \mathscr{T}_{r}=\left\{A \mid \tau_{\mathscr{F}} A=A\right\}$;

(ii) $\mathscr{T}_{h}=\mathscr{T}_{r}$ if and only if $\mathscr{F}$ is $h$-regular;

(iii) $\mathscr{T}_{n}=\mathscr{T}_{r}$ if and only if $\mathscr{F}$ is $n$-regular.

We call a map $\alpha: A \rightarrow B \mathscr{F}$-cancellable (sometimes called $\mathscr{F}$-epic), provided that for every $F \in \mathscr{F}$ and $f, g: B \rightarrow F, f \alpha=g \alpha$ implies $f=g$. We denote the smallest class containing $\mathscr{F}$ which is closed under formation of subobjects and products by $\widehat{\mathscr{F}}$, and note that this is the quotient-reflective hull of $\mathscr{F}$.

PROPOSITION 1.5. For any class $\mathscr{F}$ and any object $A, \tau \widehat{\mathscr{F}} A \leq \tau A$ and $A \in \widehat{\mathscr{F}}$ if and only if $\tau_{\mathscr{F}} A=0$. If $\mathscr{F}$ is closed under subobjects, then $A \in \widehat{\mathscr{F}}$ if and only if $\tau A=0$.

PROPOSITION 1.6. For any class $\mathscr{F}$, the following hold:

(i) the composition of $\mathscr{F}$-cancellable maps is $\mathscr{F}$-cancellable;

(ii) a map $\alpha$ is $\mathscr{F}$-dense if and only if $\operatorname{Coker}(\alpha) \in \mathscr{T}_{r}$;

(iii) every $\mathscr{F}$-cancellable map is $\mathscr{F}$-dense;

(iv) if $\mathscr{F}$ is $h$-regular, then $\mathscr{F}$-dense and $\widehat{\mathscr{F}}$-dense coincide;

(v) if $\mathscr{C}$ is additive and if $\mathscr{F}$ is closed under submodules, then $\mathscr{F}$-dense and $\mathscr{F}$-cancellable coincide.

ProOF. (i) This follows directly from the definitions.

(ii) Let $\alpha: A \rightarrow B$ have cokernel $C \in \mathscr{T}_{r}$. If $\alpha(A) \leq K$ and $K$ is $\mathscr{F}$-closed in $B$, then there is an epimorphism $g: C \rightarrow B / K$. But $B / K \in \mathscr{F}$ and $C \in \mathscr{T}_{\mathrm{r}}$ imply $B=K$ and $\alpha$ is $\mathscr{F}$-dense. 
Conversely, if $\alpha$ is $\mathscr{F}$-dense and $g: C \rightarrow D$ is a quotient map, then there is a normal subobject $K$ of $B$ containing $\alpha(A)$ with $B / K \cong D$. Thus if $B / K \in \mathscr{F}$, $K=B$ by the denseness of $\alpha$ and $D=0$. Hence $C \in \mathscr{T}_{r}$.

(iii) If $\alpha$ is $\mathscr{F}$-cancellable, $\varphi: B \rightarrow C$ is the cokernel of $\alpha$, and if $f: C \rightarrow F$ is a quotient map with $F \in \mathscr{F}$, then $0 \alpha=f \varphi \alpha$. Since $\alpha$ is $\mathscr{F}$-cancellable, $0=f \varphi$, and since $\varphi$ is surjective, $f=0$. Consequently $C \in \mathscr{T}_{r}$.

(iv) By (ii) and (iii) of Proposition $1.5, \mathscr{T}_{r}(\mathscr{F})=\mathscr{T}_{h}(\mathscr{F})=\mathscr{T}_{h}(\widehat{\mathscr{F}})$ and the result follows from (ii) above.

(v) If $\alpha$ is $\mathscr{F}$-dense, $F \in \mathscr{F}$, and $f, g: B \rightarrow F$ with $f \alpha=g \alpha$, then $K=$ $\operatorname{ker}(f-g)$ contains $\alpha(A)$ and $B / K$, being a subobject of $F$, belongs to $\mathscr{F}$. Denseness implies $K=B$ and so $f=g$.

We call an object $X \mathscr{F}$-separated provided that for every $\mathscr{F}$-dense map $\alpha: A \rightarrow B$ and pair $f, g: B \rightarrow X, f \alpha=g \alpha$ implies $f=g$. Call $X$ categorically-separated provided that for every $\mathscr{F}$-cancellable map $\alpha: A \rightarrow B$ and pair $f, g: B \rightarrow X$, $f \alpha=g \alpha$ implies $f=g$. We denote the class of all $\mathscr{F}$-separated objects by $\mathscr{F}$-Sep and the class of all categorically-separated objects by Cat-Sep. Let Diag $_{\mathscr{F}}$ denote the class of all objects $A$ for which the diagonal map $\Delta_{A}: A \rightarrow A \times A$ is $\mathscr{F}$-closed.

PROPOSITION 1.7. For any class $\mathscr{F}$ the following hold:

(i) the classes Cat-Sep and $\mathscr{F}$-Sep are closed under formation of subobjects and products, hence are quotient-reflective;

(ii) $\mathscr{F} \subset \widehat{\mathscr{F}} \subset \mathscr{F}$-Sep $\subset$ Cat-Sep;

(iii) if $\mathscr{F}$ is closed under subobjects, then $\operatorname{Diag}_{\mathscr{F}} \subset \mathscr{F}$-Sep;

(iv) if $\mathrm{Cl}(-)$ is weakly hereditary, then $\widehat{\mathscr{F}}=\mathscr{F}$-Sep $\subset \mathrm{Diag}_{\mathscr{F}}$;

(v) if $\mathscr{F}$ is closed under subobjects and $\mathrm{Cl}(-)$ is weakly hereditary, then $\left(\mathscr{T}_{h}, \widehat{\mathscr{F}}\right)$ is a generalized torsion theory. In particular, for every object $A, \tau_{\mathscr{F}}(A) \in \mathscr{T}_{h}$ and $X \in \widehat{\mathscr{F}}$ if and only if $\operatorname{Hom}_{\mathscr{C}}(A, X)=0$ for all $A \in \mathscr{T}_{h}$.

PROOF. (i) and (ii) are proved straight forwardly from the definitions.

(iii) Let $\alpha: A \rightarrow B$ be $\mathscr{F}$-dense and $f, g: B \rightarrow X$ with $f \alpha=g \alpha$ and $X \in \operatorname{Diag}_{\mathscr{F}}$. The map $\{f, g\}: B \rightarrow X \times X$ enjoys $\pi_{1} f \alpha=\pi_{2} g \alpha$ and consequently there exists a unique map $h: A \rightarrow X$ satisfying $\Delta_{X} h=\{f, g\} \alpha$. From the continuity condition and the fact that $\alpha$ is $\mathscr{F}$-dense, we have

$$
\{f, g\}(B)=\{f, g\}\left(\mathrm{Cl}_{B}(\alpha(A)) \subset \mathrm{Cl}_{X \times X}(\{f, g\} \alpha(A)) \subset X .\right.
$$

Hence there is a unique map $k: B \rightarrow X$ with $\Delta_{X} k=\{f, g\}$. From this it follows that $f=g$.

(iv) If $X \in \mathscr{F}$-Sep and $\mathrm{Cl}(-)$ is weakly hereditary, then the map $\theta: 0 \rightarrow \tau X$ is $\mathscr{F}$-dense. Thus if $\mu: \tau X \rightarrow X$ is the inclusion, then $0 \theta=\mu \theta$. Hence $\mu=0$ and 
$X \in \widehat{\mathscr{F}}$. Consider the map $\Delta_{X}: X \rightarrow X \times X$ which is the equalizer of the projections $\pi_{1}$ and $\pi_{2}$. The map $X \stackrel{e}{\rightarrow} \mathrm{Cl}_{X \times X}(X)$ is $\mathscr{F}$-dense and if $m: \mathrm{Cl}_{X \times X}(X) \rightarrow X \times X$ is the inclusion, $\pi_{1} m e=\pi_{1} \Delta_{X}=\pi_{2} \Delta_{X}=\pi_{2} m e$ implies $\pi_{1} m=\pi_{2} m$. Thus $m$ factors through $\Delta_{X}$ and $X=\mathrm{Cl}_{X \times X}(X)$.

(v) This follows from Section 8 of [1].

This result should be contrasted with the Hausdorff Characterization Theorem [13, Theorem 2.4] which shows that $\operatorname{Diag}_{\mathscr{F}}=\mathscr{F}$-Sep whenever $\mathscr{F}$ induces a weakly hereditary closure operator. Castellini [2] defines $\mathscr{F}$-Sep this way for regular closure operators, but the following examples show that the diagonal closed objects are not the correct separated objects if the closure operator is not weakly hereditary.

EXAMPLE 1.8. In the category of nilpotent groups, the class $\mathscr{F}_{\tau}$ of all torsion-free groups determines an idempotent closure operator for which $\mathscr{F}_{\tau}-\operatorname{Sep}=\mathscr{F}_{\tau}$ while $\operatorname{Diag}_{\mathscr{F}_{\tau}}$ is precisely the class of torsion-free abelian groups.

EXAMPLE 1.9. In the category of rings, either NRng, AlRng or ARng, a ring $R$ is an ideal in $R \times R$ if and only if $R$ is a zero ring.

\section{2. $H$-closedness}

Being motivated by the notion of $H$-closed topological space which generalizes the notion of topological compactness, we call an object $G H(\mathscr{F})$-closed, or more simply $H$-closed, provided that for every $F \in \mathscr{F}$ and homomorphism $f: G \rightarrow F$ with $f(G)$ normal in $F$, it follows that $F / f(G)$ belongs to $\mathscr{F}$. This notion differs from what Dikranjan and Giuli [4] call absolutely $C$-closed for a closure operator $C$, and in the same way differs from the notion of absolute $\tau$-purity for a torsion theory $\tau$ (see Golan [12]). However, the definitions are related. The important thing here is that the notion of $H$-closed encapsulates an important aspect of categorical compactness to be delineated in the next section. $H$-closed objects are always categorically compact, and it is not surprising that much of the effort in [5] and in [4] was to find conditions that forced the reverse implication, thus characterizing the categorically compacts as the $H$-closed objects. We will see that the $H$-closed objects behave in a very nice way under relatively weak assumptions on the class $\mathscr{F}$.

PROPOSITION 2.1. For any class $\mathscr{F}$, the homomorphic image of an $H$-closed object is $H$-closed.

The next theorem shows that in the case of $\mathscr{F}$ being a torsion-free class of rings, the $H$-closed rings $G$ are determined by the $H$-closedness of $G / \tau G$. 
THEOREM 2.2. For any class $\mathscr{F}$ and object $G$, consider the following statements:

(a) $\mathrm{G}$ is $\mathrm{H}$-closed;

(b) $G / \tau G$ is $H$-closed;

(c) for every $\mathscr{F}$-closed subobject $C$ of $G, G / C$ is $H$-closed.

Then:

(i) (a) implies (b) implies (c);

(ii) if $G / \tau G \in \mathscr{F}$ (for instance if $\mathscr{F}$ is closed under subdirect products), then (b) and (c) are equivalent;

(iii) if $\mathscr{F}$ is closed under normal subobjects, then (a) and (c) and hence (a), (b) and (c) are equivalent.

PrOOF. (i) and (ii) are evident, and when $\mathscr{F}$ is closed under normal subobjects, (c) implies (a) directly from the definition and Proposition 2.1 .

PROPOSITION 2.3. If $\mathscr{F}$ is closed under normal subobjects and normality is transitive for $\mathscr{F}$ objects, the class of $H$-closed objects is closed under formation of extensions and finite products.

Proof. Let $0 \rightarrow A \rightarrow B \rightarrow C \rightarrow 0$ be a short exact sequence with both $A$ and $C$ being $H$-closed. Let $F \in \mathscr{F}$ and $f: B \rightarrow F$ have $f(B)$ normal in $F$. Then $f(A)$ is normal in $f(B)$, and hence, by hypothesis, each belongs to $\mathscr{F}$ and $f(A)$ is normal in $F$. There is a commutative diagram with exact rows and columns and naturally induced maps:

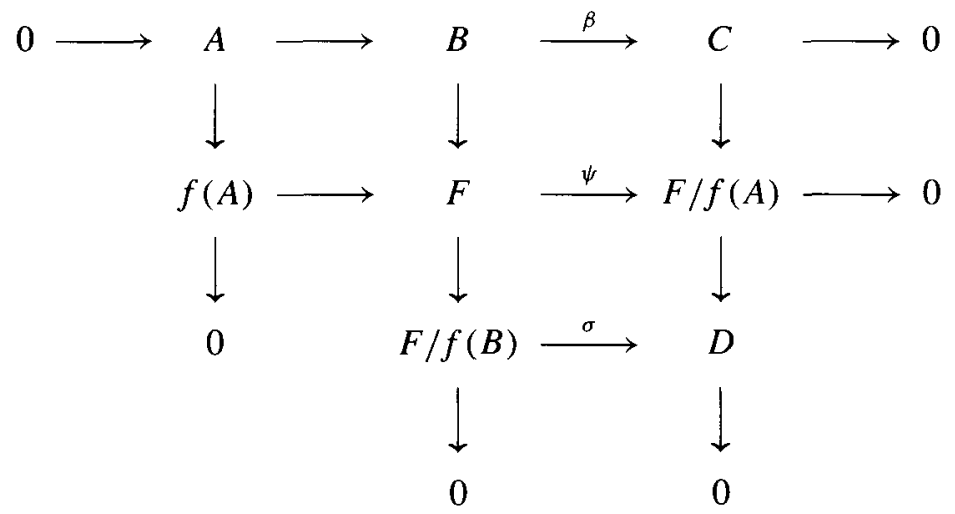

Clearly, there is an induced map $g: C \rightarrow F / f(A)$ and $g(C)=g(\beta(B))=\psi(f(B))$. Since $\psi$ is onto and $f(B)$ is normal, it follows that $g(C)$ is normal in $F / f(A)$; let $D$ denote the quotient. The induced map $\sigma$ is necessarily onto. It is a routine diagram 
chase to see that $\sigma$ is also monic. Now, $A$ being $H$-closed implies that $F / f(A) \in \mathscr{F}$, and so $C$ being $H$-closed, implies $D \in \mathscr{F}$. Thus $F / f(B) \in \mathscr{F}$ and $B$ is $H$-closed.

The module case is important enough to single out in the following.

COROLLARY 2.4. In R-Mod, if $\mathscr{F}$ is closed under subobjects, then the class of $H$-closed modules is closed under images, extensions and finite products.

PROPOSITION 2.5. In R-Mod, if $\mathscr{F}$ is closed under extensions, then the class of $H$-closed modules is $\mathscr{F}$-closed hereditary.

PROOF. Let $0 \rightarrow A \rightarrow B \rightarrow C \rightarrow 0$ be a short exact sequence with $C \in \mathscr{F}$ and let $B$ be $H$-closed. Let $F \in \mathscr{F}$ and $f: A \rightarrow F$ be a homomorphism. Consider the pushout diagram

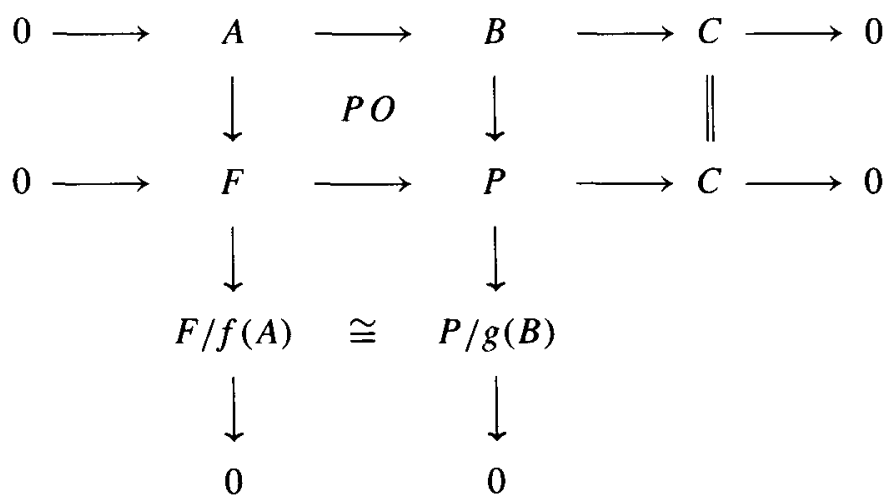

where $g: B \rightarrow P$. By hypothesis, $F, C \in \mathscr{F}$ imply $P \in \mathscr{F}$ and $B$ being $H$-closed implies $P / g(B) \in \mathscr{F}$. Thus $A$ is $H$-closed.

In R-Mod, if $\mathscr{F}=\widehat{\mathscr{F}}$, then $\mathscr{F}$ is closed under extensions if and only if $\mathscr{F}$ is the torsion-free class for a torsion theory, and in this case $\mathrm{Cl}(-)$ is weakly hereditary. For these classes then, the class of $H$-closed modules is $\mathscr{F}$-closed hereditary (see Proposition 3.1 of [4]).

In $[15,4.16]$, a conjecture related to rings was raised concerning $\mathscr{F}$-closedness. We partially answer this conjecture with the following observation.

THEOREM 2.6. If $G$ is $H$-closed and $f: G \rightarrow F$ with $f(G)$ normal in $F$, and if there exists an $\mathscr{F}$-closed subobject $C$ in $F$ with $C \leq f(G)$, then $F / f(G) \in \mathscr{F}$. 
PROOF. Consider the following commutative diagram with short exact rows and first two columns.

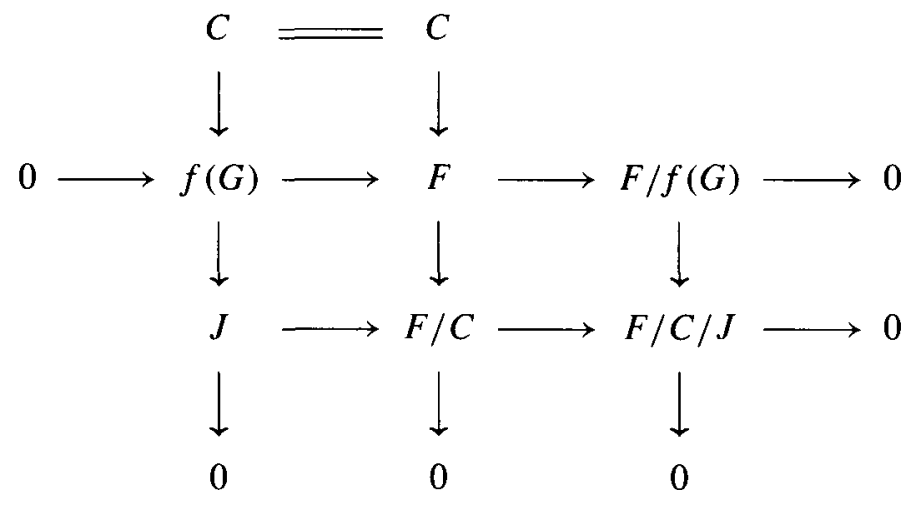

Since $G$ is $H$-closed, so are $f(C)$ and $J$. Thus $F / C \in \mathscr{F}$ implies $F / C / J \in \mathscr{F}$. A routine diagram chase (or application of an isomorphism theorem) shows that the map $F / f(G) \rightarrow F / C / J$ in the diagram is actually an isomorphism.

The following is a pleasing density theorem.

THEOREM 2.7. If $\mathscr{F}$ is closed under normal subobjects, $A$ is an $H$-closed normal subobject of an object $B$, and if $B / A \in \mathscr{T}_{r}$, then $B$ is $H$-closed.

PROOF. We apply Theorem 2.2 by showing for every $\mathscr{F}$-closed subobject $C$ of $B$, $B / C$ is $H$-closed. Let $f: B \rightarrow B / C$ be the natural map and note that $f(A)$ is normal in $B / C$. Since $A$ is $H$-closed, $D=B / C / f(A)$ belongs to $\mathscr{F}$. There is a naturally induced map $\varphi: B / A \rightarrow D$, which forces $D=0$ since $B / A \in \mathscr{T}_{r}$. So $f(A) \cong B / C$ and hence $B / C$ is $H$-closed.

The last result of this section is the routine:

PROPOSITION 2.8. For any class $\mathscr{F}$, every object is $H$-closed if and only if $\mathscr{F}$ is closed under homomorphic images.

EXAMPLE 2.9. In the category of abelian groups, multiplication by an integer $n$ determines a cohereditary non-hereditary radical. The class $\mathscr{F}(n)=\{A \mid n A=0\}$ is a variety, closed under subgroups, products, and homomorphic images (which are the only varieties of abelian groups). Thus every abelian group is $H(\mathscr{F}(n))$-closed. 


\section{3. $\mathscr{F}$-compactness}

Being motivated by the Kuratowski-Mrówka Theorem characterizing compactness for topological spaces, we say an object $G$ is $\mathscr{F}$-compact provided that for every object $H$ and $\mathscr{F}$-closed subobject $A$ of $G \times H, \pi_{2}(A)$ is an $\mathscr{F}$-closed subobject of $H$. This notion of categorical compactness was first studied by Manes [17] and later generalized by Herrlich, Salicrup, and Strecker [13] for factorization structures for morphisms. Castellini [2] developed the notion relative to a closure operator. Our results here are even a bit more general in that we do not require $\mathscr{F}$ to determine a closure operator.

PROPOSITION 3.1. For any class $\mathscr{F}$, the class of $\mathscr{F}$-compact objects is closed under formation of homomorphic images and finite products.

ProOF. Let $B$ be $\mathscr{F}$-compact and $f: B \rightarrow C$ be a cokernel map. Let $H$ be arbitrary and $A$ be $\mathscr{F}$-closed in $C \times H$. If $D$ is the inverse image of $A$ in $B \times H$, then $B \times H / D \cong C \times H / A$ and $D$ is $\mathscr{F}$-closed. The uniqueness of the (surjective, injective) factorization structure yields $\pi_{2}(D) \cong \pi_{2}(A)$. The $\mathscr{F}$-compactness of $B$ yields $\pi_{2}(A) \mathscr{F}$-closed in $H$ as desired.

The proof for finite products is even more straight forward and is omitted.

The key observation which makes the study of categorical compactness in these algebraic categories different from the study in topological categories is that the testing of the $\mathscr{F}$-closedness of $\pi_{2}(A)$ in $H$ is aided by the existence of a very natural short exact sequence which arises from the following commutative diagram with indicated pullback square having short exact rows and columns.

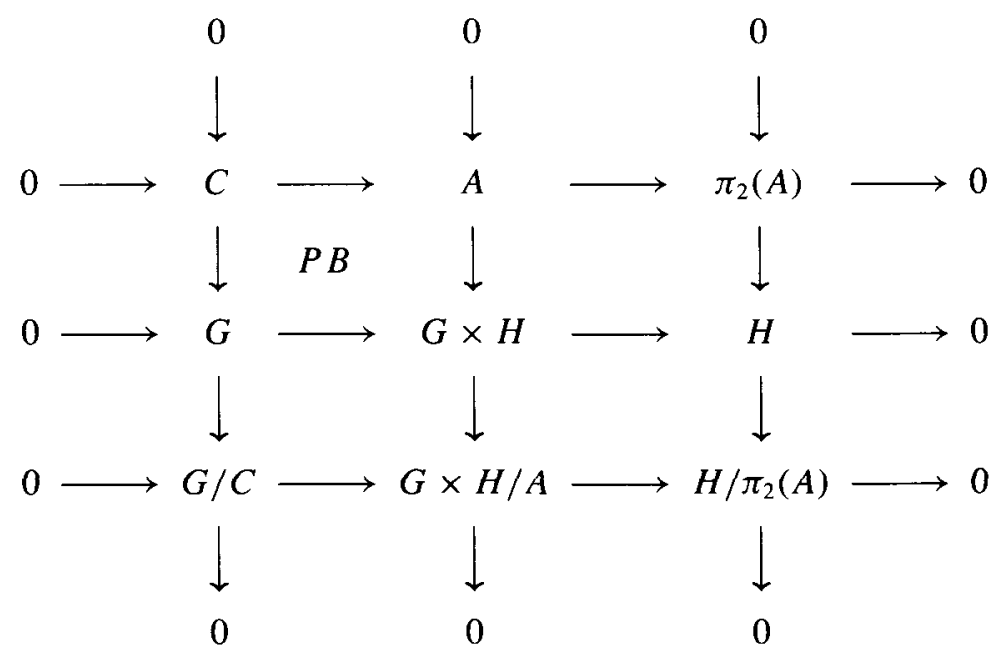


If $A$ is $\mathscr{F}$-closed in $G \times H$, the short exact sequence

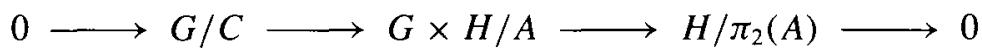

has $G \times H / A \in \mathscr{F}$ and thus the properties of $G / C$ can be used to force $H / \pi_{2}(A)$ to belong to $\mathscr{F}$ as well.

THEOREM 3.2. For any class $\mathscr{F}$ and object $G$, consider the following statements:

(a) $G$ is $\mathscr{F}$-compact;

(b) $G / \tau G$ is $\mathscr{F}$-compact;

(c) for every $\mathscr{F}$-closed subobject $C$ of $G, G / C$ is $\mathscr{F}$

Then:

(i) (a) implies (b) implies (c);

(ii) if $G / \tau G \in \mathscr{F}$ (for instance if $\mathscr{F}$ is closed under subdirect products), then (b) and (c) are equivalent;

(iii) if $\mathscr{F}$ is closed under normal subobjects, then (a) and (c) and hence (a), (b) and (c) are equivalent.

PROOF. (i) and (ii) are clear. If $\mathscr{F}$ is closed under normal subobjects, then, referring to the diagram above, $G / C$ belongs to $\mathscr{F}$ and so $G / C$ is $\mathscr{F}$-compact. Let $\varphi: G \rightarrow$ $G / C$ be the natural map and observe that the image $D=\varphi \times 1(A)$ in $G / C \times H$ is $\mathscr{F}$-closed (one needs only to observe that the quotients are isomorphic). It follows that $\pi_{2}(D) \cong \pi_{2}(A)$ and the $\mathscr{F}$-compactness of $G / C$ implies $\pi_{2}(A)$ is $\mathscr{F}$-closed in $H$.

The next result is immediate and was the motivation behind the notion of $H$-closed.

THEOREM 3.3. For any class $\mathscr{F}$

(i) an $H$-closed object is $\mathscr{F}$-compact;

(ii) iffor every homomorphism $f: G \rightarrow F$ with $f(G)$ normal in $F$ and $F \in \mathscr{F}$, the graph morphism $\{1, f\}: G \rightarrow G \times F$ is $\mathscr{F}$-closed, then $G$ being $\mathscr{F}$ compact implies $G$ is $H$-closed.

PROOF. The proof of (i) follows directly from the above diagram and the exactness of $0 \rightarrow G / C \rightarrow G \times H / A \rightarrow H / \pi_{2}(A) \rightarrow 0$. Statement (ii) follows from the definition of $\mathscr{F}$-compactness and the fact that $G$ being $\mathscr{F}$-closed in $G \times F$ implies that $f(G)$ is $\mathscr{F}$-closed in $F$.

The next two results shed light on [5, Proposition 4.5] and [4, Corollary 5.8]. 
COROLLARY 3.4. If $\mathscr{F}$ is quotient-reflective and $\mathrm{Cl}(-)$ is weakly hereditary, then $G$ is $\mathscr{F}$-compact if and only if $\mathrm{G}$ is $\mathrm{H}$-closed.

Proof. The hypothesis on $\mathscr{F}$ implies that $\mathscr{F}=\widehat{\mathscr{F}}=\mathscr{F}$-Sep $=\operatorname{Diag}_{\mathscr{F}}$. By Proposition 1.13 of [2], $Y \in \operatorname{Diag}_{\mathscr{F}}$ if and only if for every $X$ and map $f: X \rightarrow Y$, the graph map $\{1, f\}: X \rightarrow X \times Y$ is $\mathscr{F}$-closed. Thus the hypothesis of Theorem 3.3 (ii) is satisfied.

COROllaRY 3.5. In R-Mod, $G$ is $H$-closed if and only if $G$ is $\mathscr{F}$-compact.

The following proposition is a slight restatement of [4, Corollary 5.5 (b)] and [2, Theorem 1.8]. Recall that if $\mathrm{Cl}(-)$ is a weakly hereditary idempotent closure operator, then the composition of $\mathscr{F}$-closed maps is $\mathscr{F}$-closed.

PROPOSITION 3.6. If the composition of $\mathscr{F}$-closed maps is $\mathscr{F}$-closed, then the class of $\mathscr{F}$-compact objects is closed under formation of $\mathscr{F}$-closed subobjects.

The following is an immediate consequence of Proposition 2.8 and Theorem 3.3.

PROPOSITION 3.7. If $\mathscr{F}$ is closed under images, then every object is $\mathscr{F}$-compact.

EXAMPLE 3.8. The following examples are from group theory. Examples involving rings will be given in the next section.

(a) In the category of all groups, if $\mathscr{F}$ is the class of all groups, then $\mathrm{Cl}(-)$ is simply the normal closure and all groups are $\mathscr{F}$-compact.

(b) In the category of abelian groups, the class $\mathscr{F}_{\tau}$ of all torsion-free groups determines a weakly hereditary idempotent closure operator. A group $G$ is $\mathscr{F}_{\tau^{-}}$ compact if and only if $G$ is $H$-closed if and only if $G / \tau G$ is divisible. Here $\tau G$ denotes the maximal torsion subgroup of $G$. See [5] and [4].

(c) In the category of locally nilpotent groups, the class $\mathscr{F}_{\tau}$ of all torsion-free locally nilpotent groups determines an idempotent closure operator which is not weakly hereditary. However, a locally nilpotent group $G$ is $\mathscr{F}_{\tau}$-compact if and only if $G$ is $H$-closed if and only if $G / \tau G$ is radicable. (A group is radicable provided that for every element $g$ and $n>0$, there is a solution to the equation $g=x^{n}$.) See [6].

(d) Example 2.9 shows every abelian group to be $\mathscr{F}(n)$-compact.

(e) In the category of nilpotent groups, let $\mathscr{F}$ be the class of all reduced groups. Every nilpotent group $G$ contains a maximal normal complete subgroup $d G$, and $G \in \mathscr{F}$ if and only if $d G=0$. A nilpotent group $G$ is $\mathscr{F}$-compact if and only if $G$ is $H$-closed, if and only if $G / d G$ is cotorsion [9].

(f) In the category of abelian groups, let $\mathscr{F}_{p}=\left\{G \mid p^{\omega} G=0\right\}$. A group $G$ is $\mathscr{F}_{p}$-compact if and only if $G / p^{\omega} G$ is topologically compact in its $p$-adic topology [7]. 
(g) In the category of nilpotent groups, let $\mathscr{F}$ be the class of all torsion-free $p$ reduced groups. A group $G$ is $\mathscr{F}$-compact if and only if $G / \tau G$ is $p$-divisible [7].

\section{Categorical compactness for rings}

In this section we apply the preceding development to the category of not necessarily associative rings NRng and to the category of associative rings ARng. In doing so, we extend some results of Gardner [11] for associative rings. As has been already noted, the $H$-closed objects and the $\mathscr{F}$-compact objects are well-behaved when the class $\mathscr{F}$ is closed under subdirect products and normal subobjects. Semisimple and torsion-free classes of rings are closed under subdirect products and torsion-free classes are closed under ideals. Perhaps most interestingly, the class of usual torsion-free rings (that is, the underlying abelian group is torsion-free in the usual sense) is a torsion-free class closed under subrings, products, and extensions which gives rise to an idempotent closure operator which is not weakly hereditary. The categorically compact rings with respect to this class behave in a degenerate way in the sense that the analogues of the well-known topological theorems - closed subspaces of compact spaces are compact and compact subspaces of Hausdorff spaces are closed - both fail. The analogues do hold for the group theoretic examples given in the previous section. Unless stated otherwise, all rings will be considered to be objects of NRng.

THEOREM 4.1. For any class $\mathscr{F}$ closed under direct summands, if $R$ contains a left (or right) identity, then $R$ is $\mathscr{F}$-compact.

PROOF. Let $R$ and $H$ be arbitrary rings and $A$ be an ideal in $R \times H$. If $(a, b) \in A$, then since $1 \in R,(1,0)(a, b)=(a, 0) \in A$ and so $(0, b) \in A$. Hence $A=$ $\pi_{1}(A) \times \pi_{2}(A)$ and $R \times H / A \cong R / \pi_{1}(A) \times H / \pi_{2}(A)$.

Modifying slightly the approach of Dikranjan and Giuli [4], if $\mathscr{A}$ and $\mathscr{B}$ are classes of rings, a ring $G$ is called $(\mathscr{A}, \mathscr{B})$-compact provided that for every ring $H \in \mathscr{B}$ and $\mathscr{A}$-closed ideal $A \leq G \times H, H / \pi_{2}(A) \in \mathscr{A}$. The proof of Theorem 4.1 can be trivially adapted to show:

THEOREM 4.2. For any class $\mathscr{F}$ closed under direct summands, all rings are (F, Rng $\left.^{1}\right)$-compact, where $\mathrm{Rng}^{1}$ denotes the class of all rings with a right or left identity.

Recall that a semisimple class of rings is an $n$-regular class closed under formation of subdirect products and extensions. A torsion-free class is a semisimple class that is closed under ideals. Examples distinguishing between these concepts are well-known; see for example [16]. 
Results 4.1 and 4.2 can be generalized for associative rings, showing that many rings are always $\mathscr{F}$-compact. Following Roos [18], we can produce various regularities for associative rings as follows. Let $G=\left\{g_{1}, g_{2}, \cdots, g_{n}\right\}$ be a set of at least two integral polynomials. For each ring $R$ define $G_{R}(r)=g_{1}(r) R g_{2}(r) R \cdots R g_{n}(r)$, for every element $r \in R$. Then $\left\{G_{R}: R\right.$ is an associative ring $\}$ is a regularity ([18, Theorem 5]), called a polynomial regularity or p-regularity. The ring $R$ is called $G$-regular if $r \in G_{R}(r)$ for each $r \in R$. If at least one of the $g_{i} \in G$ has zero constant term, then the $G$-regularity is called nullic. Let $S_{R}(r)=\Sigma\left\{G_{R}^{i}(r): i \in I\right\}$ where each $G_{R}^{i}$ is a $p$-regularity chosen in such a way that $S_{R}(r)$ is an ideal of $R$ for each $r \in R$. Then $S_{R}(r)$ determines a regularity, called a summable p-regularity or $s p$-regularity and $R$ is called $S$-regular if $r \in S(r)$ for each $r \in R$. Roos gives a list of eleven well-known regularities, all of which are $p$ - or $s p$-regularities, and these include $D$-regularity, von Neumann regularity, $\lambda$-regularity, left quasi-regularity, left pseudo-regularity, strongly regular rings, and Brown-McCoy radical rings.

THEOREM 4.3. In ARng let $\mathscr{F}$ be any class which is closed under direct summands. For any ring $R$ let $S_{R}(r)=\sum\left\{G_{R}^{i}(r): i \in I\right\}$ where each $G_{R}^{i}$ is a nullic p-regularity chosen in such a way that $S_{R}(r)$ is an ideal of $R$ for each $r \in R$. If $R$ is $S$-regular, then $R$ is $\mathscr{F}$-compact.

ProOF. Let $R$ be $S$-regular, $H$ be arbitrary, and $A$ be an ideal in $R \times H$. If $(a, b) \in A$, then $a \in S_{R}(a)$, and so for some $k \in \mathbb{Z}, j=1, \cdots, k$, we have $a=\sum_{j} s_{j} a t_{j}$, where (for each $j$ ) either $s_{j}$ and $t_{j} \in R$ or $s_{j}=1 \in \mathbb{Z}$ and $t_{j} \in R$ or $s_{j} \in R$ and $t_{j}=1 \in \mathbb{Z}$. Now identify $(1,0)(a, b)$ or $(a, b)(1,0)$ with $(a, b)$ where necessary and observe that

$$
\begin{aligned}
\sum\left(s_{j}, 0\right)(a, b)\left(t_{j}, 0\right) & =\left(\sum s_{j} a t_{j}, 0\right) \\
& =(a, 0) \in A .
\end{aligned}
$$

It follows that $(0, b) \in A$. Thus $A=\pi_{1}(A) \times \pi_{2}(A)$ and $R \times H / A \cong R / \pi_{1}(A) \times$ $H / \pi_{2}(A)$.

The following result is proved in a similar way.

THEOREM 4.4. In ARng, for any class $\mathscr{F}$ which is closed under direct summands, all rings are (F, S-Reg)-compact, where $S$-Reg denotes the class of all $S$-regular rings described in Theorem 4.3 .

The next result for associative rings extends [11, Proposition 3.1] and shows that most of the well-known semisimple classes (for example, rings with trivial Jacobsen radical, nil-radical, or prime radical) do not yield interesting compact classes. 
THEOREM 4.5. In ARng, if $\mathscr{F}$ is semisimple and consists solely of semiprime rings, then every ring is $\mathscr{F}$-compact.

PROOF. Gardner [11] showed that for a semisimple class $\mathscr{F}$ associated with a radical class $\mathscr{R}$, the $\mathscr{F}$-closed maps are closed under composition if and only if $\mathscr{R}$ is supernilpotent. This occurs if and only if $\mathscr{F}$ consists of semiprime rings.

In this case, the ring of integers $\mathbb{Z}$ belongs to $\mathscr{F}$ and hence if $R^{1}$ denotes the ring with identity obtained from the ring $R$ be adjoining 1 in the usual fashion, then $R$ is an $\mathscr{F}$-closed ideal in $R^{1}$. If $H$ is an arbitrary ring, then $R \times H$ is $\mathscr{F}$-closed in $R^{1} \times H$. Thus if $A$ is an $\mathscr{F}$-closed ideal in $R \times H, A$ is also $\mathscr{F}$-closed in $R^{1} \times H$, and the $\mathscr{F}$-compactness of $R^{1}$ implies that $\pi_{2}(A)$ is $\mathscr{F}$-closed in $H$.

There is an interesting situation for the class of (usual) torsion-free rings discussed in [15] and [16]. For any ring $R$, the set of torsion elements $\tau R$ form an ideal, and $R / \tau R$ is torsion-free. We let $\mathscr{T}_{\tau}$ and $\mathscr{F}_{\tau}$ denote the classes of all torsion and torsion-free rings respectively.

LEMMA 4.6. [16] The pair $\left(\mathscr{T}_{\tau}, \mathscr{F}_{\tau}\right)$ is a hereditary torsion theory for the category NRng.

Thus $\mathscr{F}_{\tau}$ is a torsion-free class of rings for NRng. The class of $\mathscr{F}_{\tau}$-compact rings is non-trivial and behaves degenerately as mentioned in the introduction to this section. Since $\mathscr{F}_{\tau}$ is closed under direct summands, Theorems 4.3 and 4.4 apply, showing that for associative rings ARng, $G$-regular rings are $\mathscr{F}_{\tau}$-compact for nullic $G$-regularities. Our next observation is that the idempotent closure operator induced by $\mathscr{F}_{\tau}$ is not weakly hereditary; for if it were, then it would also be when restricted to associative rings. By Gardner's results, this would force $\mathscr{F}_{r} \cap$ ARng to consist solely of semiprime rings which is false.

By Theorem 4.1 , the ring of integers $\mathbb{Z}$ is $\mathscr{F}_{\tau}$-compact. However, the zero ring over the integers $\mathbb{Z}^{0}$ is not $\mathscr{F}_{\tau}$-compact. Our next result explains this behavior.

THEOREM 4.7. Let $R$ be a ring.

(i) If $R^{+} / \tau R^{+}$is divisible as an abelian group, then $R$ is $\mathscr{F}_{\tau}$-compact; consequently, every Artinian ring and every regular ring is $\mathscr{F}_{\tau}$-compact.

(ii) If $R$ is a zero ring, then $R$ is $\mathscr{F}_{\tau}$-compact if and only if $R^{+} / \tau R^{+}$is divisible as an abelian group. Thus not all rings are $\mathscr{F}_{r}$-compact.

PROOF. (i) is clear. The statements about artinian and regular rings follow from the description of their underlying groups ([10, Vol. II]).

(ii) If $R$ is a zero ring which is $\mathscr{F}_{\tau}$-compact, then without loss of generality, assume $R$ is torsion-free (see 3.2). If $R^{+}$, the additive group of $R$, is not divisible, let $\widehat{R}$ denote 
the divisible hull of $R$ considered as a zero ring. Then $R$ is an ideal in $\widehat{R}$ and $\widehat{R} / R$ is torsion. This contradicts the $\mathscr{F}_{\tau}$-compactness of $R$ as $R$ is $\mathscr{F}_{\tau}$-closed in $R \times \widehat{R}$.

PROPOSITION 4.8. If $R$ is an ideal in a ring with identity $S$ and $S / R$ is torsion, then $R$ is $\mathscr{F}_{\tau}$-compact.

PROOF. For arbitrary $H$, let $A$ be an $\mathscr{F}_{\tau}$-closed ideal in $R \times H$ and let $(a, b) \in A$. Since $n \in R$ for some $n \in \mathbb{Z},(n, 0) \in R \times H$ and so $(n a, 0) \in A$. But $(n a, 0)=$ $n(a, 0)$, and the torsion-freeness of $R \times H / A$ implies $(a, 0) \in A$. Hence $(0, b) \in A$, so $A \cong \pi_{1}(A) \times \pi_{2}(A)$ and it follows that $H / \pi_{2}(A)$ is torsion-free.

The following examples show that the topological analogues discussed in the introduction fail to hold despite the class $\mathscr{F}_{\tau}$ being closed under subrings, products, and extensions.

EXAMPLE 4.9. (a) Let $\mathbb{Z}^{0}$ be the zero ring on the integers and let $\mathbb{Z}^{1}$ be the ring obtained from $\mathbb{Z}^{0}$ by adjoining an identity in the usual fashion. Then $\mathbb{Z}^{0}$ is an ideal in $\mathbb{Z}^{1}$ and $\mathbb{Z}^{1} / \mathbb{Z}^{0} \cong \mathbb{Z}$. Thus $\mathbb{Z}^{1}$ is $\mathscr{F}_{\tau}$-compact and $\mathbb{Z}^{0}$ is $\mathscr{F}_{\tau}$-closed in $\mathbb{Z}^{1}$, but is not $\mathscr{F}_{\tau}$-compact.

(b) As a ring itself, $n \mathbb{Z}$ is $\mathscr{F}_{\tau}$-compact by Proposition 4.8 and it is an ideal in $\mathbb{Z}$. The ring $\mathbb{Z}$ belongs to $\mathscr{F}_{\tau} \subset \mathscr{F}_{\tau}$-Sep, but $\mathbb{Z} / n \mathbb{Z}$ being torsion shows that $n \mathbb{Z}$ is not $\mathscr{F}_{\tau}$-closed in $\mathbb{Z}$. Hence $n \mathbb{Z}$ is not $H$-closed, so that the class of $H$-closed rings is properly contained in the class of $\mathscr{F}$-compact rings. This also answers in the negative the second part of [15, Conjecture 4.16].

\section{Acknowledgements}

The first author wishes to express his gratitude for the support received from the Departments of Mathematics of the Technikon Pretoria and the University of South Africa, where this work was conducted, and for financial aid from the FRD.

We wish to thank Leon van Wyk for alerting us to the work of C. Roos.

\section{References}

[1] C. Cassidy, M. Hébert and G. M. Kelly, 'Reflective subcategories, localizations and factorization systems', J. Austral. Math. Soc. (Series A) 38 (1985), 287-329.

[2] G. Castellini, 'Compact objects, surjectivity of epimorphisms and compactifications', Cahiers Topologie Géom. Différentielle Catégoriques XXXI-1 (1990), 53-65.

[3] D. Dikranjan and E. Giuli, 'Closure operators I', Topology Appl. 27 (1987), 129-143. 
[4] - 'Factorizations, injectivity and compactness in categories of modules', Comm. Algebra 19 (1991), 45-83.

[5] T. H. Fay, 'Compact modules', Comm. Algebra 16 (1988), 1209-1219.

[6] —_, 'Remarks on the Mal'cev completion of torsion-free locally nilpotent groups', Cahiers Topologie Géom. Différentielle Catégoriques XXXV-1 (1994), 75-84.

[7] T. H. Fay and M. J. Schoeman, 'The torsion completion, the $p$-adic completion, and categorical compactness for nilpotent groups', in preparation.

[8] T. H. Fay and G. L. Walls, 'A characterization of categorically compact locally nilpotent groups', Comm. Algebra, to appear.

[9] — , 'Completions and categorical compactness for nilpotent groups', Quaestiones Math., to appear.

[10] L. Fuchs, Infinite abelian groups I, II (Academic Press, New York, 1973).

[11] B. J. Gardner, 'Radicals which define factorization systems', Comment. Math. Univ. Carolin. 32 (1991), 601-607.

[12] J. S. Golan, Torsion theories, Pitman Monographs and Surveys in Pure and Applied Math. (Longman Scientific and Technical, Harlow, 1986).

[13] H. Herrlich, G. Salicrup and G. E. Strecker, 'Factorizations, denseness, separation, and relatively compact objects', Topology Appl. 27 (1987), 157-169.

[14] H-J. Hoehnke, 'Radikale in allegemeinen algebren', Math. Nachr. 32 (1966), 347-383.

[15] S. V. Joubert, 'Categorically compact rings and modules', Chinese J. Math. 20 (1992), 347-365.

[16] W. G. Leavitt and R. Wiegandt, 'Torsion theory for not necessarily associative rings', Rocky Mountain J. Math. 9 (1979), 259-271.

[17] E. G. Manes, 'Compact Hausdorff objects', Topology Appl. 4 (1979), 341-360.

[18] C. Roos, 'A class of regularities for rings', J. Austral. Math. Soc. (Series A) 27 (1979), 437-453.

The University of Southern Mississippi

Hattiesburg, MS

USA
The Technikon Pretoria

Pretoria West 0001

South Africa 